\title{
Targeting and Scheduling Problem for Fleld Artillery
}

\author{
Ojeong Kwon, Kyungsik Lee and Sungsoo Park \\ Dept. of Industrial Engineering, Korea Advanced Institute of Science and Technology \\ 373-1 Gusong-dong,Yusong-gu,Taejon 305-701 Korea
}

\begin{abstract}
We consider the targeting and the fire sequencing problem for field artillery. We show that the targeting problem, which can be modeled as a problem with nonlinear constraints, can be transformed into a set of independent bounded variable knapsack problems. We also propose a mathematical model for the fire sequencing problem which is NP-hard and developed a heuristic to solve the problem. Computational results using randomly generated data are presented. (C) 1997 Elsevier Science Ltd
\end{abstract}

Keyword : Targeting, Scheduling, Artillery

\section{Introduction}

We consider the targeting and the fire sequencing problem for filed artillery. Targeting Problem(TP) is to allocate weapon systems to targets so that desired target destroy level is achieved with minimum cost. Other objectives and/or constraints may be considered for the problem. Once the weapon systems are allocated to targets, we need to schedule the firing sequence of the weapon systems on targets so that firing can be finished as soon as possible. When we plan the targeting operation, we have to take into account tactical mission, ability of weapon system(range and deflection), desired target destroy level, available ammunition amount, target characteristics and so on.

The targeting problems have been considered by many researchers for recent several decades. Flood[1] formulated (TP) as a problem of minimizing enemy threat with nonlinear objective function and linear constraints. Danzig[7] modified Flood's model with reliability concept and approached it by means of separable convex programming approximation. Bracken and McCormick[2] considered (TP) with nonlinear objective function and general assignment constraints and they also considered the mathematical model for inflicting specified damage with linear objective cost. They applied Linear Programming to solve the latter problem and rounded the fractional solutions to nearest integers. Ash[1] soived Flood's assignment model consisted of small kill probability by Lagrangian multiplie method. Manne[7] transformed Flood's model into a transportation problem approximately and solved it. Recently, Sherali, Kim and Parrish[11] suggested 3 kinds of linear functions that provide the lower bound on the nonlinear objective function for probabilistic partial set covering problem which has a similar nonlinear objective function structure with (TP).
Here we seek an optimal strategy to allocate weapon systems to the targets achieving desired target destroy level with minimum cost. Target destroy level is given as the probability of destroying the target. Cost can be determined by integrating various costs(ammunition cost, delivery cost, opportunity cost, and cost derived from other factors). This model can provide the guidance to determine the amounts of ammunition to be prepared for an operation and how to assign various weapon systems to targets according to tactical mission, combat boundary, and limited resources

Once we get optimal weapon allocation results, we will be faced with the question of how to establish the firing sequence to achieve minimum time span, which we call the Fire Sequencing Problem(FSP). Short and intensive impact on the targets achieves the maximum effectiveness of surprise. Moreover, shorter time span enables the operation to be more flexible. The two problems may be considered together as one model. However, combined model will be too complex to solve. Moreover, there are possibilities that the operation officers can get weapon-target allocations using other mathematical models and/or subjective judgments, in which case it will be more desirable to consider the problems separately.

We present the formulations of (TP) and (FSP) and analyze these problems in section 2 . We also show that (TP) can be solved in pseudo-polynomial time and note that (FSP) is NP-hard. In section 3, we present a heuristic method to solve (FSP) which solves the node packing problems[8] repeatedly. We report computationsl results with randomly generated data in section 4 . Finally, we give concluding remarks in section 5 . 


\section{Problem Description}

\section{(1) Targeting Problem}

To formulate the targeting problem, we use the unit duration concept. The unit duration is defined as the minimum firing interval to synchronize the heterogeneous weapon systems. Joint Munitions Effectiveness Manual(JMEM) provides the damage probability according to target characteristics, the types of munitions, the posture of target, impact angle, radius of target, and so on[12].

We use the following notations.

$W$ : Set of weapon systems

$T:$ Set of targets

$A$ : Set of arcs, where $(i, j) \in A$ if and only if weapon $i$ can fire on target $j$

$x_{i i}$ : Decision variable to denote the number of unit duration of weapon $i$ assigned to target $j$

$c_{i j}$ : Firing cost of weapon $i$ to target $j$ during one unit duration

$p_{i i}$ : The probability of destroying target $j$ by one unit duration of fire from weapon $i, 0<p_{i j}<1$

$d_{j}$ : The minimum desired probability of destroying target $j$. $0<d_{1}<1$

$u_{i j}$ : Upper bound on the number of unit duration firing from weapon $i$ to target $j$

The weapon-target fire ability graph $K=(W, T, A)$ is a bipartite graph in which each weapon and target corresponds to a node. The fire ability state from weapon $i$ to target $j$ corresponds to the edge $(i, j)$. Due to the ability of weapons, combat organization and combat boundary, $K$ is generally sparse in a real situation. Then, (TP) can be formulated as follows.

$$
\begin{aligned}
& \text { (TP) } \min \sum_{(i, j) \in A} c_{i j} x_{i j} \\
& \text { s.t } 1-\quad \Pi\left(1-p_{i j}\right)^{x_{1}} \geq d_{j} \text {, for all } j \in T \\
& \{(i \in M(i, j) \in A\} \\
& x_{i j} \leq u_{i j} \text {, for all }(i, j) \in A \\
& x_{i j} \text { nonnegative integer, for all }(i, j) \in A
\end{aligned}
$$

Constraints (1) imply that the probability of destroying target $j$ should be greater than or equal to the minimum desired level. These nonlinear constraints can be transformed into linear inequalities by taking logarithm on both sides. This transformation does not change the feasible region of (TP). Therefore, we get another problem which is equivalent to (TP)

$$
\begin{aligned}
& \text { (TP1) } \quad \min \sum c_{i j} x_{i j} \\
& \text { s.t } \sum_{\{(i \leq W(i, j) \in A\}}^{m} a_{i j} x_{i j} \geq b_{1} \text {, for all } j \in T \\
& x_{i j} \leq u_{i j} \text {, for all }(i, j) \in A \\
& x_{i j} \geq 0 \text { integers for all }(i, j) \in A \\
& \text { where, } 0<a_{i f}=-\ln \left(1-p_{i j}\right) \quad 0<b_{j}=-\ln \left(1-d_{j}\right)
\end{aligned}
$$

The coefficients in constraints ( 3 ) are not rational numbers However, by multiplying proper integers on both sides, we can approximate the feasible region of (TP) to the desired accuracy. (TP1) can be decomposed into subproblems each of which corresponds to a target. Each subproblem is a bounded variable knapsack problem which can be solved by a dynamic programming algorithm with a running time $O\left(|W| b_{j}{ }^{2}\right)[8]$.

\section{(2) Fire Sequencing Problem}

After we get optimal allocation of firing duration from weapons to targets, we have to decide the sequence of firing. Due to the characteristics of military operations, (FSP) has a few features which can be different from the scheduling problem arising in the manufacturing environments. First, a weapon can fire on only one target at a time. Second, if a weapon starts firing against a target, it should terminate firing after consuming all planned duration. If a weapon changes the target before consuming all planned duration, the additional set up time would increase the time span and decrease the effectiveness of surprise. Considered from the target side, the fire starting time on a target from all assigned weapons should be the same. The reason is to achieve tactical surprise. Surprise is a principle of strategy, operational art and tactics. However, the fire terminating time of each weapon on a target may be different.

To formulate (FSP), let us define the followings

$W$ : Set of weapons

$T$ : Set of targets

$t_{1}$ : Decision variable to denote the fire starting time on target $j$, for all $j \in T$

$t_{f}$ : Decision variable to denote the fire starting time on a dummy target which is to be fired on last $p_{i j}$ : Planned firing duration from weapon $i$ to target $j$

$W(j, k)$ : Set of weapons which need to fire on both target $j$ and $k$

(FSP) $\min t$

$$
\begin{aligned}
& \text { s.t } t_{1} \geq t_{i}+\max _{i \in W}\left(p_{i j}\right) \text {, for all } j \in T \\
& t_{j}-t_{k} \leq-\max _{i \in W(j, k)}\left(p_{i j}\right)+M\left(1-x_{j k}\right) \text {, for } j<k \\
& t_{k}-t_{j} \leq-\max _{i \in W(j, k)}\left(p_{i k}\right)+M\left(x_{j k}\right), \quad \text { for } j<k \\
& t_{i} \in Z_{+} \text {for } i \in T \\
& x_{j k} \in\{0,1\} \text {, for all } j, k \in T, j<k \\
& \text { where, } M \text { is a large number }
\end{aligned}
$$

The binary variable $x_{j k}$ is equal to 1 if and only if target $j$ precedes target $k$ in the firing sequence, for all j,k $\in T$, $j<k$. (FSP) has $|T|+1$ general integer variables and $|T|(|T|-1) / 2$ binary variables and $|T|^{2}$ constraints. Constraints (4) mean that the dummy target's starting time is the latest and we wish to minimize it. Constraints (5) and (6) are the disjunctive constraints which represent the starting time relationship between any two targets. It is possible that more than one target can be fired on at the same time if they do not share a common weapon that is supposed to fire on them. If $W(j, k)=\varnothing$, then $-\max _{i \in W(j, k)}\left(p_{i j}\right)$ and $-\max _{i \in W(j, k)}\left(p_{i k}\right)$ will be 0 and, by constraints $(5)$ and $(6)$, $t_{1}=t_{k}$ can be accomplished.

It can be shown that the decision problem of (FSP) is strongly NP-complete and remains strongly NP-complete 
even if the number of weapons is two[6].

In the manufacturing systems, the jobs are usually processed by only one machine at a time. A problem similar to (FSP) in the manufacturing systems setting is the Simultaneous Resource Scheduling Problem(SRSP), in which it is allowed that a machining operation may use multiple resources simultaneously. In the early days, attention is given on (SRSP) in the computer and communication systems area[4], but recent research on (SRSP) is more focused on manufacturing systems. Dobson and Karmarkar [4] suggested a Lagrangian relaxation and surrogate relaxation for (SRSP) to find a lower bound on the optimal value.

We can regard (FSP) as a job sequencing problem in the manufacturing system: The machines correspond to the weapons and the jobs to the targets. In this regard, (FSP) is similar to (SRSP). However, while (FSP) permits that the finishing time of an operation on the machines can be different, (SRSP) does not. Furthermore, while the objective of (SRSP) is to minimize total weighted flow time, the objective of (FSP) is to minimize time span.

Another important characteristics of (FSP) is the history dependent property. When sequencing a job(target), its starting time depends on the whole sequence of the jobs scheduled so far. This property distinguishes (FSP) from the well-known Scheduling Problem with Sequence Dependent Set-up times(SPSDS). In the latter case, the starting time of a job depends only on the job scheduled just before it and so the problem can be modeled by the Traveling Salesman Problem(TSP)[9]. However, the history dependent property of (FSP) makes it impossible to use the traveling salesman problem.

\section{Heuristic Method for (FSP)}

Since (FSP) is NP-hard, we developed a heuristic method to solve the problem. Given a specific time slot, we allocate targets to the time slot so that as many as weapon systems can fire on the assigned targets. This procedure is repeated until all targets are scheduled. Hence the method is a greedy type heuristic. Given a specific time $s$ in the entire time horizon, let $N(s)$ be the set of targets which need to be fired on at time $s$. We define the independent targets in $N(s)$ as the set of targets in $N(s)$ such that any two of them do not share a common weapon. Then the independent targets can be fired on at the same time. Our strategy is to find independent targets in $N(s)$ and schedule them at time $s$ so that the utilization of all the weapon system is high.

Let $G(s)=(N(s), E(s))$ be a graph where $N(s)$ is the set of nodes denoting the set of targets which need to be fired on at time $s$ and $E(s)$ be the set of edges joining the nodes in $N(s)$. An edge $(i, j)$ is in $E(s)$ if and only if target $i$ and $j$ share a common weapon, i.e., they cannot be fired on at the same time. Let $n_{j}, j \in N(s)$ be the weight of node $j$ which is given as the number of weapons that need to fire on target $j$. Then our problem is to find a maximum weight node packing in $G(s)$, which is known to be NP-hard[5]. Therefore, we also used a greedy type heuristic to solve the problem. At first, the node weights are sorted in nonincreasing order. The node at the top of the list is removed and added to the Temporary Node Packing Set(TNPS) as long as the addition of the node to TNPS does not destroy the node packing property of the nodes in TNPS. At the end of the procedure, the nodes in TNPS constitute a node packing. Detailed description of the overall procedure for (FSP) is given as follows.

Let $R$ be a $|W| \times M$ matrix where $M$ is a large integer. $R$ is used to record the firing schedule. We set $R(i, s)=j$ if, at time $s$, target $j$ is scheduled to be fired on by weapon $i$. We set $R(i, s)=0$ if weapon $i$ does not fire on any target at times.

\section{Heuristic for FSP}

Initialize) $s=1$, TNPS $=\varnothing, R(i, t)=0$, for all $i \in W, t=1, \ldots, M$.

Step 1) Termination condition check

If $p_{i j}=0$, for all $i \in W, j \in T$.

Print time table $\mathrm{R}$ and Stop.

Step 2) Find the independent targets at time $s$ :

2.1) Construct a graph $G(s)=(N(s), E(s))$, where $N(s)=\left\{j \in T \mid p_{i j}>0\right.$, for some $\left.i \in W\right\}$

$E(s)=\left\{(i, j) \mid p_{k i}>0, p_{k j}>0\right.$, for some $\left.k \in W, i, j \in T\right\}$

Let $n_{j}$ be a weight of $j \in N(s)$, where

$n_{j}=\left|W_{i}\right|, W_{i}=\left\{i \in W \mid p_{i j}>0\right\}$

2.2)If $\{j \in N(s)\}(i, j) \notin E(s)$, for all $i \in T N P S\}=\varnothing$,

then go to step 3 .

select a node(target)

$j=\operatorname{argmax}_{j \in N(s)}\left\{n_{j} \mid(i, j) \notin E(s)\right.$, for all $\left.i \in T N P S\right\}$

2.3) TNPS $\leftarrow$ TNPS $\cup\{j\}$, go to 2.2 .

Step 3) Updating procedure :

Let $\vec{p}^{\cdot}=\min _{j \in \text { TNPS }}\left\{p_{i j} \mid p_{i j}>0, i \in W\right\}$

3.1) For each $i \in W$, if $p_{i j}>0$ for some $j \in$ TNPS,

$R(i, t)=j$ for $t=s, \ldots, s+p^{*}-1$

3.2) $p_{i j} \leftarrow p_{i j}-p^{\cdot}$ for all $i \in W_{1} j \in T N P S$, such that $p_{i j}>0$

3.3) Let $V(s)$ be the subset of TNPS where $j \in V(s)$, if updated $p_{i j}=0$ for all $i \in W$ TNPS $\leftarrow$ TNPS $\backslash \mathrm{V}(s)$

3.4) $s \leftarrow s+p^{\circ}$ go to step 1 .

In the above algorithm, at each time we get a TNPS, at least one $p_{i j}$ is set to 0 and the time to get a TNPS in step 2 is $O\left(|T|^{2}\right)$. Therefore, the above algorithm runs in $O\left(|W| \times|T|^{3}\right)$

\section{Computational Results}

We consider proper size of weapons and targets. The battery is the basic echelon of field artillery for targeting in real situation. We set the number of batteries to be $3-20$ and the number of targets is set to about 3-5 times the number of batteries, considering tactical mission and combat boundaries. Considered weapon-target pairs are shown in table 1 . The battery, battalion, regiment and brigade echelon of artillery plan the targeting operations for corresponding maneuver unit support. Therefore, we set the number of weapons considering the echelon and rein- 
forcing artillery units.

To test (TP), we randomly generated data. We generated 10 test problems for each weapon, target pair. To reflect reality, the damage probabilities were randomly generated in the interval $[0.1,0.2]$ and the desired damage probabilities of the targets were randomly taken from $[0.3,0.5]$. We took logarithms on the randomly generated data and they were rounded to the nearest integers after each number is multiplied by 100 . All problems could be solved within 0.5 CPU seconds on a HP9000/715(50MHz) workstation by a dynamic programming algorithm.

Based on the outputs from (TP), we tested both the heuristic method(HM) and the integer programming(IP) approach for the fire sequencing problem. The integer programming formulation was solved by CPLEX 3.0. Test results are summarized in table 1 . For small problems $(3 \times 10,20 \times 5,20 \times 10)$, the integer program could find optimal solutions. However, for the remaining problems, it could not find optimal solutions within the prescribed time limits (1800 seconds). In the last column of table 1 , the average lower/upper bound of the problems are given, which are expressed as percentages of the optimal values. The size of the integer programming formulation depends on the number of targets. Hence it takes more time to solve the problem as the number of targets becomes large. On the other hand, the heuristic method could find optimal solutions for all test problems in a very short time. We note that the quantity $\max _{i \in W}\left(\Sigma_{j \in T} p_{i j}\right)$ provides a lower bound on the optimal value. The solutions obtained by heuristic were compared with the lower bounds and it validated that the solutions were all optimal.

Table. 1 Summary of comparison for (HM) \& (IP)

\begin{tabular}{ccc}
\hline $\begin{array}{c}\text { Problem Size } \\
\text { Weapon } \times \text { Target) }\end{array}$ & $\begin{array}{c}\text { Mean CPU Time } \\
\text { (HM/IP)(Second) }\end{array}$ & $\begin{array}{c}\text { Mean(Lower/Upper)Bound } \\
\text { of (IP)[\% to optimum] }\end{array}$ \\
\hline $3 \times 10$ & $0.0 / 324.1$ & All optimal \\
$3 \times 15$ & $0.0 / * *$ & $74.5 / 103.1$ \\
$3 \times 20$ & $0.0 / * *$ & $47.4 / 114.2$ \\
$12 \times 30$ & $0.1 / * *$ & $66.7 / 118.3$ \\
$12 \times 50$ & $0.4 / * *$ & $41.3 / 178.6$ \\
$12 \times 70$ & $1.0 / * *$ & $32.6 / 187.3$ \\
$20 \times 60$ & $0.9 / * *$ & $45.2 / 181.0$ \\
$20 \times 80$ & $1.9 / * *$ & $31.6 / 207.2$ \\
$20 \times 100$ & $3.21 * *$ & $26.7 / 159.3$ \\
$20 \times 5$ & $0.0 / 0.02$ & All optimal \\
$20 \times 10$ & $0.0 / 14.0$ & All optimal \\
$20 \times 15$ & $0.0 / * *$ & $94.2 / 101.2$ \\
\hline
\end{tabular}

1800 seconds time limit

We need a little more remarks on the performance of the heuristic method. As we mentioned earlier, the weapontarget fire ability graph is sparse in general. After solving (TP), the obtained weapon-target assignment graph is even sparser. Therefore, we can schedule many targets at the same time, which makes it possible to schedule many tasks within the time span given by the lower bound stated above. If the weapon-target assignment graph is dense, there will be a few independent targets and any arbitrary sequence of the targets may yield a near optimal solution. Therefore it is expected that the medium density problem will be the most difficult one to solve.

\section{Conclusions}

We proposed models and algorithms for the targeting problem and the fire sequencing problem. In real situations, there may exist other constraints and/or objectives to be considered. The model may be changed reflecting these conditions. One of the important characteristics of the problems we consider is that they need to be solved quickly. We showed that the proposed algorithms, when applied to real problems, could find good solutions in a very short time.

If there exists a time span limitation for the fire sequencing problem, the model may not yield a feasible solution. In that case, we may need to trade-off between the cost of assigning targets and the time needed to finish firing. It needs further research in this respect. Also there may be the case that some targets need to be fired on at a preassigned time. Those targets should be allocated first in the required time slot when we solve (FSP). Hence (FSP) can be regarded as a special case of that problem.

The Republic of Korea Army(ROKA) has been developing TACtical FIRE(TACFIRE) system which enables all the firing procedure become automatic from observation to firing. When TACFIRE system is accomplished, (ROKA) can control and combine all fire systems more effectively. We think that (TP) and (FSP) heuristic algorithms will play important roles as submodules in TACFIRE system for field antillery and will contribute to the computerization and automation of the fire control system.

\section{References}

1. Ash, M., 1959, Flood's Assignment Model for Small Kill Levels, Operations Research, : 258-260.

2. Bracken, J., and McCormick, G., 1980, Selected Application of Nonlinear Programming, John Wiley \& Sons, New York.

3. DARCOM-P-706-101, 1977, Enqineering Design Handbook Army Weapon Systems Analysis, Alexandria

4. Dobson, G., and Karmarkar, U., 1989, Simultaneous Resource Scheduling to Minimize Weighted Flow Times, Operations Research, 37, : 592-600

5. Garey, M., and Johnson, D., 1979, Computers and Intractability. Bell Telephone Laboratories, Incorporated, New Jersey.

6. Lee, K., Kwon, O., Park, K., Park, S., 1996, Complexity of the Fire Sequencing Problem, Submitted to Discrete Applied Mathematics.

7. Manne, A., 1958, A Target Assignment Problem, Operations Research, 5-6, : 346-351.

8. Nemhauser, G., and Wolsey, L., 1988, Integer and Combinatorial Optimization, John Wiley \& Sons, New York.

9. Pinedo, M., 1995, Scheduling. Theory Algorithms and Systems, Prentice Hall, New Jersey

10. ROKA HQ, 1979, FM 6-40 Field Artillery tactics.

11. Sherali, H., Kim S., Parrish, E., 1991, Probabilistic Partial Set Covering Problem, Naval Research Logistics, 41-51

12. 61JTCG/ME-E-KS-77-14, 1988, Programmable Calculator Manual for Evaluating Effectiveness of Nonlinear Surface-to-Surface Indirect Fire Weapons against Area Targets. 